\title{
Nitric Oxide-Stimulated Increase in Extracellular Adenosine Accumulation in Rat Forebrain Neurons in Culture Is Associated with ATP Hydrolysis and Inhibition of Adenosine Kinase Activity
}

\author{
Paul A. Rosenberg, Ya Li, Minou Le, and Yumin Zhang, \\ Department of Neurology and Program in Neuroscience, Children's Hospital and Harvard Medical School, Boston, \\ Massachusetts 02115
}

\begin{abstract}
Adenosine is a putative endogenous sleep-inducing substance, and nitric oxide has been implicated in arousal and sleep mechanisms. We found that various nitric oxide donors, including diethylamine NONOate (DEA/NO), stimulated large increases in extracellular adenosine in nearly pure cultures of forebrain neurons. The effect of DEA/NO could be blocked by 2-phenyl4,4,5,5-tetramethyl-imidazoline-1-oxyl-oxide and could not be mimicked by degraded solutions of DEA/NO or by DEA itself; therefore, it was caused by nitric oxide release on hydrolysis of the parent compound. The accumulation of adenosine was not blocked by probenecid or GMP, suggesting that neither extracellular CAMP nor extracellular AMP was the source, and that adenosine was therefore the most likely species transported across the plasma membrane. To pursue this further, we tested the effect of DEA/NO on cellular ATP and found a significant fall
\end{abstract}

in ATP associated with exposure to nitric oxide. In addition, exposure to DEA/NO nearly completely inhibited adenosine kinase activity. It has been found previously that adenosine kinase is inhibited by its substrate, adenosine. We found that exposure to nitric oxide increased intracellular adenosine to $125 \pm 18 \%$ of control values $(p<0.01)$, consistent with the possibility that in our system the inhibition of adenosine kinase is related to an increase in intracellular adenosine, and that the effect of nitric oxide on extracellular adenosine is significantly potentiated by substrate inhibition of adenosine kinase. Furthermore, nitric oxide-stimulated adenosine accumulation may be important in the regulation of behavioral state.

Key words: nitric oxide; adenosine; sleep; adenosine kinase; ATP; laterodorsal tegmental nucleus; pedunculopontine tegmental nucleus
Nitric oxide, discovered by Furchgott and colleagues (Furchgott and Zawadzki, 1980; Furchgott, 1998) to be an intercellular signaling molecule, appears to be involved in long-term potentiation (Schuman and Madison, 1994) and signal processing mechanisms (Kara and Friedlander, 1999). Other functions for nitric oxide in the brain are likely. Nitric oxide synthase (NOS) is richly expressed in a nuclear group that plays an important role in arousal, the lateral dorsal tegmental (LDT)/pedunculopontine tegmental nucleus (PPT), suggesting a possible role in sleep physiology. Nitric oxide synthase is also found in cholinergic nuclei of the basal forebrain implicated in sleep mechanisms, including the septum and nuclei of the diagonal band, and in the hypothalamus (Vincent and Kimura, 1992).

Several studies using inhibitors of nitric oxide synthase have shown inhibition of sleep with blockade of enzyme activity in the rabbit (Kapas et al., 1994b) and the rat (Kapas et al., 1994a; Dzoljic et al., 1996; Burlet et al., 1999). In contrast to these studies that suggest that nitric oxide facilitates the production of sleep, other studies have shown that release of nitric oxide in the thalamus is associated with the waking state and REM sleep (Burlet and Cespuglio, 1997; Williams et al., 1997; Cespuglio et al., 1998). This is consistent with patterns of activity of the NOS containing LDT/ PPT neurons, which are most active in waking and in REM sleep. Furthermore, nitric oxide has been shown to have effects on neurons in the thalamus (Pape and Mager, 1992) and medial pontine reticular formation (Leonard and Lydic, 1997), consistent with a role in the production of waking and REM sleep.

Received March 21, 2000; revised May 15, 2000; accepted June 6, 2000.

This work was supported by a grant from National Institutes of Health (HL59595) and a Mental Retardation Research Center grant to Children's Hospital. We thank Drs. Clifford Saper, Thomas Scammell, David White, Woody Weiss, and, in particular, Robert Greene of the Harvard Center on Sleep Neurobiology and Sleep Apnea for helpful discussions and encouragement.

Correspondence should be addressed to Dr. Paul A. Rosenberg, Department of Neurology, Children's Hospital, 300 Longwood Avenue, Boston, MA 02115. E-mail: rosenberg@a1.tch.harvard.edu.

Copyright (C) 2000 Society for Neuroscience $0270-6474 / 00 / 206294-08 \$ 15.00 / 0$
Adenosine appears to be involved in the regulation of behavioral state (Rainnie et al., 1994; Portas et al., 1997). It was found using microdialysis techniques that adenosine accumulated in the basal forebrain of cats during waking, increased with sleep deprivation, and decreased when animals were allowed to sleep. Inf usion of an inhibitor of adenosine uptake caused an increase in sleep when the dialysis probe was located in the basal forebrain but not in the thalamus (Porkka-Heiskanen et al., 1997). Thus, adenosine appears to be an endogenous sleep-inducing substance, with regionally specific actions.

We do not know how adenosine levels are regulated in the brain. This knowledge would enable us to understand how behavioral state is regulated. We have previously used dissociated cell cultures containing both neurons and astrocytes derived from rat embryonic forebrain to study mechanisms of extracellular adenosine accumulation (Rosenberg and Dichter, 1989; Rosenberg and Li, 1994, 1995). In the present study, we used neuronal cultures derived from rat forebrain to investigate the effect of nitric oxide on extracellular adenosine accumulation and found that nitric oxide is a potent and efficacious stimulator of adenosine release from neurons. These findings suggest the possibility that nitric oxide release in target areas of the nitrergic neurons of the LDT/PPT might itself promote the accumulation of extracellular adenosine.

An abstract has been published previously (Li and Rosenberg, 1998).

\section{MATERIALS AND METHODS}

Tissue culture. Neuronal cultures were prepared from embryonic day 16 Sprague Dawley rat fetuses using methods similar to those previously described but modified to produce cultures that contained $<1 \%$ astrocytes (Rosenberg, 1991; Wang et al., 1998). Cultures were initially plated on poly-L-lysine-coated 24-well plastic plates (Costar, Cambridge, MA) using an 80:10:10 (v/v) mixture of DMEM (11960-010; Life Technologies, Grand Island, NY), Ham's F-12 (N-4888; Sigma, St. Louis, MO), heatinactivated iron-supplemented calf serum (HyClone A2151), containing 2 $\mathrm{mm}$ glutamine, $25 \mathrm{mM}$ HEPES, $24 \mathrm{U} / \mathrm{ml}$ penicillin, and $24 \mu \mathrm{g} / \mathrm{ml}$ streptomycin in a $5 \% \mathrm{CO}_{2}$ (balance air) incubator at $36^{\circ} \mathrm{C}$. Cell proliferation was inhibited by exposure to $5 \mu \mathrm{M}$ cytosine arabinoside at $24 \mathrm{hr}$ in vitro for 72 
hr. On the fourth day of culture, the medium was completely removed and replaced with 90\% MEM, 10\% NuSerum IV (Collaborative Research), 2 $\mathrm{mm}$ glutamine, $5 \mathrm{~mm}$ HEPES, containing $10 \mu \mathrm{g} / \mathrm{ml}$ superoxide dismutase (Boehringer Mannheim, Indianapolis, IN), $1 \mu \mathrm{g} / \mathrm{ml}$ catalase (Sigma CV40), total glucose $11 \mathrm{~mm}$, and total sodium bicarbonate $9.3 \mathrm{~mm}$, plus $2 \%$ B27 supplement (Life Technologies 17504-036). Medium was not subsequently changed. To prevent evaporation of water, culture dishes were kept on "wet dishes" containing filter paper that was always kept wet. Protein was $98 \pm 19 \mu \mathrm{g} / \mathrm{well}(n=11)$ determined with a bovine serum albumin standard using bicinchoninic acid (Pierce, Rockford, IL).

HPLC. Adenosine assay was performed exactly as described previously to measure all adenine-containing compounds in the extracellular medium (Rosenberg and $\mathrm{Li}, 1994)$. In brief, samples and standards were derivatized using chloracetaldehyde to produce the fluorescent etheno compounds; detection was accomplished using a McPherson fluorimeter with the 265 $\mathrm{nm}$ mercury emission band of a $200 \mathrm{~W}$ xenon-mercury lamp. All samples were run with an internal standard, and ratios of peak heights in the samples to the peak height of the internal standard were compared with the same for standards. Adenosine was measured in 50 or $100 \mu \mathrm{l}$ aliquots of extracellular medium applied to the column, and these data are used for the figures herein.

Experimental design: exposure to nitric oxide donors. In all such experiments, cultures in 24-well plates were first washed three times in HBSS containing $0.1 \%$ bovine serum albumin, and then they were placed in Earle's Balanced Salt Solution (EBSS) and preincubated for $2 \mathrm{hr}$. At zero time, nitric oxide donors were added from a $100 \times$ stock in $10 \mathrm{~mm} \mathrm{NaOH}$. At the desired time, medium was removed and placed in a microcentrifuge tube containing EDTA to yield a final concentration of $10 \mathrm{~mm}$. Samples were derivatized the day of the experiment and stored at $4^{\circ} \mathrm{C}$ until assay, which was within $7 \mathrm{~d}$.

Experimental design: assay of adenosine kinase activity. For assay of adenosine kinase, the procedure of Lynch et al. (1998) was followed closely, with minor modification. Adenosine kinase activity of neuronal cultures was determined by measuring the rate of phosphorylation of ${ }^{14} \mathrm{C}$-adenosine. For this purpose, [U- $\left.{ }^{14} \mathrm{C}\right]$-adenosine $[489 \mathrm{mCi} / \mathrm{mmol} ; \mathrm{Am}$ ersham (CFB.54), Arlington Heights, IL] was used. Cultures were treated exactly as they were when testing for the effects of nitric oxide donors on extracellular adenosine accumulation with respect to the initial wash and preincubation. Cultures were exposed to nitric oxide donor for $5 \mathrm{~min}$, and then radioactivity was added $(0.04 \mu \mathrm{M}$ adenosine final concentration). A selected intervals, medium was removed, and $200 \mu \mathrm{l}$ of ice-cold $20 \mathrm{~mm}$ sodium acetate solution, $\mathrm{pH} 4.0$, with $2 \mathrm{mM}$ EDTA, was added to each well. The cells were immediately lysed by freeze-thawing with ethanol-dry ice, and the cell lysate was spotted onto ion exchange disks (Whatman DE-81; Fisher Scientific, Pittsburgh, PA). The disks were then washed with $2 \mathrm{~mm}$ ammonium formate, rinsed successively with distilled water, methanol, and acetone, dried in room air, and exposed to a solution of $0.1 \mathrm{M} \mathrm{HCl} / 0.4 \mathrm{M}$ $\mathrm{KCl}$, and bound radioactivity was measured by liquid scintillation.

Experimental design: assay of intracellular ATP. For assay of ATP, the Bioluminescent Somatic Cell Assay kit from Sigma (stock FL-ASC) was used. Cultures were treated exactly as they were when testing for the effects of nitric oxide donors on extracellular adenosine accumulation with respect to the initial wash and preincubation. At selected time points after addition of drug or vehicle, medium was removed, 100 or $200 \mu \mathrm{l}$ of releasing agent was added, cultures were agitated briefly, another equal volume of water was added, and plate was placed on wet ice until assay. ATP assay mix was used at a 25 -fold dilution, and $100 \mu l$ of the extract was assayed.

Experimental design: assay of intracellular adenine containing compounds by HPLC. For the assay of ATP, AMP, and adenosine, we used an extraction method similar to that used for the adenosine kinase procedure described above. Cultures were exposed to diethylamine NONOate (DEA/ NO) for $5 \mathrm{~min}$. The extracellular media was removed, and then an ice-cold solution of $20 \mathrm{~mm}$ sodium acetate, $\mathrm{pH} 4.0$, with $2 \mathrm{~mm}$ EDTA, was added to the cells. Cells were immediately lysed by freeze-thawing with ethanol-dry ice. The lysate was spun for $10 \mathrm{~min}\left(14,000 \mathrm{rpm}, 4^{\circ} \mathrm{C}\right)$. The supernatant was then collected and derivatized for HPLC analysis.

Statistics. Statistical significance was assessed using ANOVA with the Tukey-Kramer post hoc multiple comparison test. Statistical analysis was performed using the Instat program from GraphPad Software (San Diego, CA). All experiments were repeated a minimum of three times. In general, experiments were performed with three to four replicate samples, and error bars represent intra-assay variation expressed as the SD. In Figures, the following convention is used for indicating statistical significance: ${ }^{*} p<$ $0.05 ; * * p<0.01 ; * * * p<0.001$

All NONOate compounds were obtained from Cayman Chemical Co. (Ann Arbor, MI). Other compounds were obtained from Sigma. Tissue culture media were obtained from Life Technologies unless noted otherwise. Purified bovine hemoglobin was obtained as a $100 \mathrm{mg} / \mathrm{ml}$ solution from Biopure Corporation (Cambridge, MA) and was aliquoted and stored at $-80^{\circ} \mathrm{C}$. HPLC analysis of this hemoglobin solution revealed the presence of low concentrations of AMP. Therefore, in experiments with hemoglobin, GMP $500 \mu \mathrm{M}$ was included to prevent hydrolysis of AMP from this source to adenosine, once it had been established (see Results) that GMP had no effect on DEA/NO-evoked adenosine accumulation.

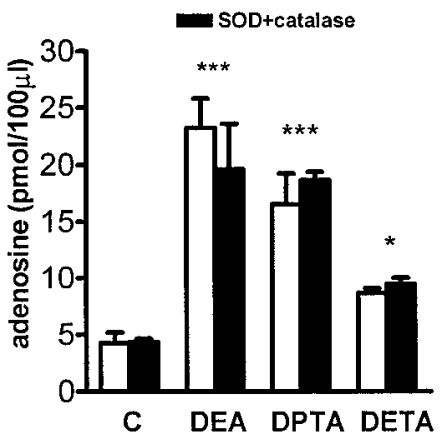

Figure 1. Nitric oxide donors evoke extracellular adenosine accumulation. Rat forebrain neuron cultures were exposed to DEA/NO $(D E A), \mathrm{DPT} / \mathrm{NO}$ $(D P T A)$, or DETA/NO $(D E T A)$ in the presence and absence of superoxide dismutase $(250 \mathrm{U} / \mathrm{ml})$ and catalase $(1000 \mathrm{U} / \mathrm{ml})$ at $1 \mathrm{~mm}$ for $30 \mathrm{~min}$, after which medium was removed and assayed for adenosine. DEA/NO, DPT/ NO, and DETA/NO produced significant increases in adenosine compared with control. There was no effect of the presence of superoxide dismutase and catalase. The experiment shown is representative of three experiments that were performed.

\section{RESULTS}

\section{Effect of nitric oxide donors}

In our initial experiments, we exposed cultures of forebrain neurons to a series of nucleophile/nitric oxide adducts (Maragos et al., 1991) with half-lives (at $37^{\circ} \mathrm{C}$ in aqueous solution at physiological $\mathrm{pH}$ ) of $2.1 \mathrm{~min}$ for DEA/NO (Maragos et al., 1991), $3 \mathrm{hr}$ for dipropylenetriamine NONOate (DPT/NO), and $20 \mathrm{hr}$ for diethylaminetriamine NONOate (DETA/NO) (Mooradian et al., 1995). In these experiments, nitric oxide donors were tested at $1 \mathrm{~mm}$, and superoxide dismutase and catalase were included in some experimental categories because of evidence that nitric oxide is toxic to neurons and that the toxicity is attributable to peroxynitrite (Lipton et al., 1993), formed by the reaction of nitric oxide and superoxide. The presence of superoxide dismutase and catalase, however, did not have a significant effect on the accumulation of adenosine evoked by the nitric oxide donors. Figure 1 shows a representative experiment. DEA/NO, DPT/NO, and DETA/NO all produced a significant increase in adenosine in the extracellular medium. In three experiments, we found increases of $627 \pm 157$, $443 \pm 8$, and $265 \pm 59 \%$, respectively, with DEA/NO, DPT/NO, and DETA/NO. Thus, the adenosine accumulation observed was inversely related to the half-life of the donors, as would be expected, because for a given incubation period, a donor with a half-life much shorter than that period (DEA/NO) will have released all its nitric oxide, whereas a donor with a half-life much longer (DETA/NO) will have released only a small fraction of its nitric oxide.

Because nitric oxide is known to be toxic to neurons, a major concern was whether the adenosine that we measured in the extracellular medium was released from injured cells, rather than by a specific process activated by nitric oxide. We excluded this possibility on several grounds. If accumulation of adenosine in the extracellular medium were caused by plasma membrane damage and nonspecific leak of intracellular adenosine into the extracellular space, then we would expect to see other adenine compounds as well. However, the chromatograms of medium from cultures treated with nitric oxide donors showed only a peak corresponding to adenosine. Plasma membrane injury would also be expected to result in the release of lactate dehydrogenase (LDH). However, we found that there was no significant LDH release from DEA/NOtreated cultures over the time course of the experiments $(n=2)$. Finally, the lack of effect of superoxide dismutase and catalase suggested that the effect was not caused by peroxynitrite formation (Koppenol et al., 1992) and not dependent on cell injury, because others have shown that the toxicity of nitric oxide to neurons depends on the formation of peroxynitrite (Lipton et al., 1993).

The fact that the stimulation of adenosine accumulation was 


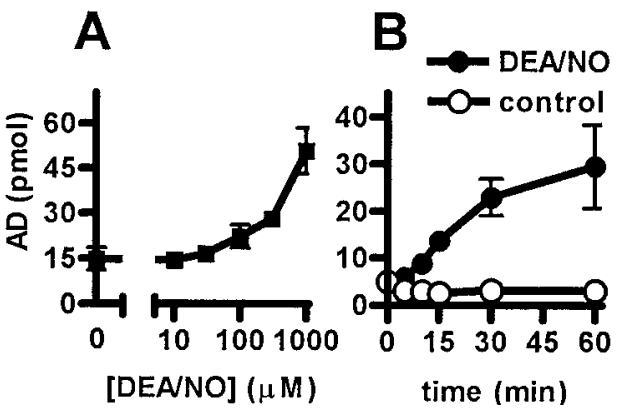

Figure 2. Concentration and time dependence of the effect of DEA/NO on adenosine accumulation. $A$, Neuronal cultures were exposed to DEA/NO at selected concentrations up to $1000 \mu \mathrm{M}$ for $30 \mathrm{~min}$, after which medium was removed and assayed for adenosine, reported here in pmol/100 $\mu \mathrm{l}$ aliquots for both $A$ and $B$. There was a concentration-dependent increase in adenosine concentration in the extracellular medium, but the process did not saturate at $1 \mathrm{~mm}$. $B$, DEA/NO was added to neuronal cultures, and medium was sampled at selected times and assayed for adenosine. DEA/NO caused a time-dependent increase in extracellular adenosine concentration, reaching a maximum by $30 \mathrm{~min}$. The experiments shown are representative of four experiments that were performed.

observed with different donors suggested that the effect was caused by nitric oxide and was not from the base released on hydrolysis in solution. For most subsequent experiments, we chose to use DEA/ NO. Because it has a short half-life, with this donor exposures are to a pulse of nitric oxide of short duration (minutes). No effect on extracellular adenosine was observed when cultures were exposed to DEA, the base produced on decomposition of DEA/NO, and no effect was observed when solutions containing DEA/NO were preincubated 20-24 hr at room temperature, allowing decomposition of the DEA/NO before exposure to cultures $(n=3$; data not shown).

\section{Concentration dependence}

The relationship of the concentration of DEA/NO to the extracellular adenosine attained after a 30 min incubation was examined using selected concentrations up to $1 \mathrm{~mm}$. Figure $2 A$ shows a representative experiment. In four experiments, the response was not saturating by $1 \mathrm{~mm}$.

\section{Time course}

The time dependence of the effect of DEA/NO was examined (Fig. $2 B$ ). In three experiments, large increases in extracellular adenosine were observed, reaching a maximum by $30 \mathrm{~min}$. Therefore, in most subsequent experiments, to obtain a near maximal response, cultures were exposed to DEA/NO for a 30 min interval, and a concentration of $100 \mu \mathrm{M}$ was used. Figure $2 B$ shows a representative experiment.

\section{Mediation by nitric oxide}

To confirm that the effect of DEA/NO was caused by nitric oxide, we used 2-phenyl-4,4,5,5,-tetramethyl-imidazoline-1-oxyl-3-oxide (PTIO), a scavenger that reacts directly and stoichiometrically with nitric oxide (Maeda et al., 1995). Figure 3 shows a representative experiment of three that were performed. PTIO at $100 \mu \mathrm{M}$ completely blocked the extracellular accumulation of adenosine evoked by $100 \mu \mathrm{M}$ DEA/NO.

\section{Source of extracellular adenosine}

There are several routes by which extracellular adenosine may be increased, including by extracellular degradation of ATP, coreleased by exocytosis with a number of neurotransmitters and neuromodulators (Stone et al., 1990), by degradation of extracellular cAMP transported out of all cells that accumulate cAMP in response to activation of adenylyl cyclase (Barber and Butcher, 1983; Rosenberg and Dichter, 1989; Rosenberg and Li, 1994), and by transport of adenosine itself across the plasma membrane (Baldwin et al., 1999). The last step in the degradation of ATP and cAMP to adenosine is the hydrolysis of AMP to adenosine by

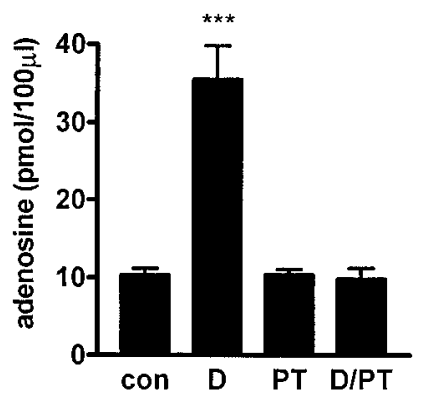

Figure 3. PTIO inhibits the formation of extracellular adenosine evoked by DEA/NO. The nitric oxide scavenger PTIO $(P T ; 100 \mu \mathrm{M})$ completely blocked the effect of DEA/NO $(D ; 100 \mu \mathrm{M})$ on extracellular adenosine accumulation. The experiment shown is representative of three experiments that were performed.

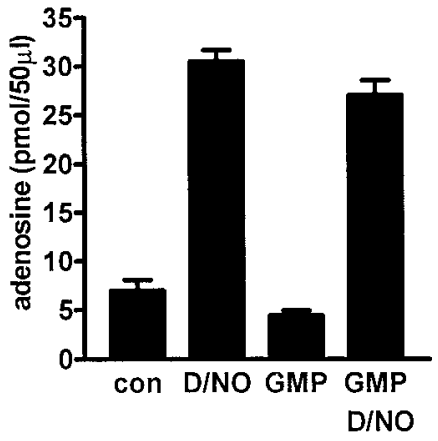

Figure 4. DEA/NO-stimulated adenosine accumulation is resistant to the 5 '-nucleotidase inhibitor guanosine monophosphate. Cultures were exposed to DEA/NO $(D / N O ; 100 \mu \mathrm{M})$ with or without $500 \mu \mathrm{M}$ GMP for 30 min. The presence of GMP had no significant effect on the accumulation of adenosine or on the basal level of adenosine. The experiment shown is a representative experiment of four that were performed.

5'-ectonucleotidase. This enzyme is inhibited by GMP (MacDonald and White, 1985; Pearson, 1987; Rosenberg and Li, 1995, 1996); therefore, if an increase in extracellular adenosine derives from extracellular ATP or cAMP, GMP should block it. We found previously that $5 \mathrm{mM}$ GMP completely blocked cAMP-derived adenosine accumulation evoked by forskolin or vasoactive intestinal peptide in mixed cultures of astrocytes and neurons (Rosenberg and Li, 1995, 1996). However, as has been noted before (MacDonald and White, 1985), use of such a high concentration of GMP is associated with numerous peaks in the chromatogram attributable to impurities in the GMP and the high sensitivity of HPLC. Therefore, we used a lower concentration, $500 \mu \mathrm{M}$, which was found in previous studies to block $61 \pm 5 \%(n=4)$ of the extracellular adenosine accumulation evoked by $100 \mu \mathrm{M}$ forskolin in neuronal cultures similar to those used in the present study (P. A. Rosenberg and Y. Li, unpublished observations). It was found, however, that $500 \mu \mathrm{M}$ GMP had no effect on DEA/NO-stimulated adenosine accumulation. Figure 4 shows a representative experiment of four that were performed. Finally, in previous studies, we found that cAMP-derived adenosine accumulation was blocked by probenecid and by the cyclic nucleotide phosphodiesterase inhibitor RO 201724 (Rosenberg and Li, 1994), but these had no effect on DEA/ NO-evoked adenosine accumulation ( $n=2$; data not shown). Therefore, this accumulation of adenosine appeared to be caused by the transport of adenosine itself across the plasma membrane.

Seven transporters of adenosine are now known: two that are equilibrative and five that are concentrative (Thorn and Jarvis, 1996; Yao et al., 1997; Baldwin et al., 1999). The two equilibrative transporters in the rat are characterized as either sensitive or resistant to pharmacological inhibition using nitrothiobenzylinosine (NBTI) below $1 \mu \mathrm{M}$. Four of the five concentrative transporters are resistant to pharmacological inhibition, with only a small degree of blockade observed at $100 \mu \mathrm{M}$. We tested NBTI and 


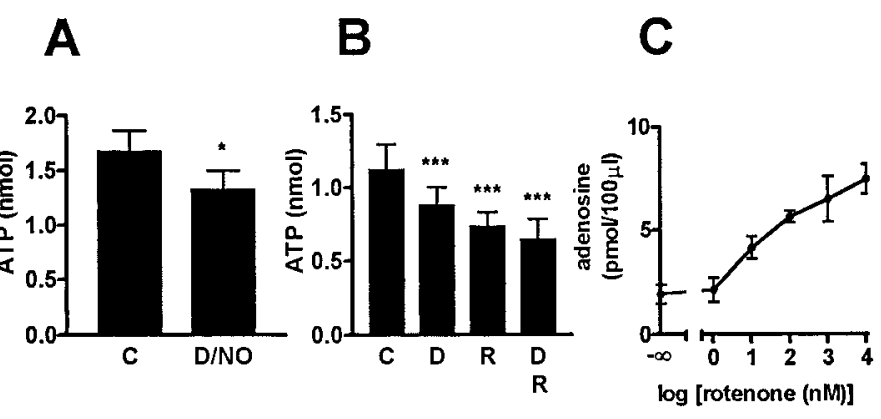

Figure 5. Effect of DEA/NO and rotenone on intracellular ATP content. $A$, Cultures were preincubated for $2 \mathrm{hr}$ as in all previous experiments in EBSS, after which they were exposed to DEA/NO $(D / N O)$ at $100 \mu \mathrm{M}$ or no drug $(C)$ for $30 \mathrm{~min}$. Cellular ATP was measured by firefly luciferase chemiluminescence. The data shown are pooled from nine experiments. DEA/NO produced a significant $(p<0.05)$ decrease in cellular ATP content to $\sim 80 \%$ of control value. $B$, The effects on cellular ATP of rotenone $(R ; 100 \mathrm{nM})$ and $\operatorname{DEA} / \mathrm{NO}(D ; 100 \mu \mathrm{M})$ were compared. Cultures were exposed to DEA/NO or rotenone, or both, for $30 \mathrm{~min}$, after which cellular ATP was assayed. Rotenone and DEA/NO both produced a significant $(p<0.001)$ decrease in cellular ATP content. DEA/NO and rotenone together did not produce a larger decrease than rotenone alone. The figure shows data pooled from three experiments. $C$, Cultures were exposed to rotenone at selected concentrations for $30 \mathrm{~min}$, after which medium was collected and assayed for adenosine. Rotenone potently stimulated extracellular adenosine accumulation. The experiment shown is representative of three that were performed.

dipyridamole for their ability to block the egress of adenosine from neuronal cultures treated with DEA/NO. No significant decrease was observed with up to $100 \mu \mathrm{M}$ of each drug $(n=3$; data not shown).

\section{Nitric oxide-induced adenosine accumulation is cGMP independent}

An important effect of nitric oxide in signal transduction is the activation of soluble guanylyl cyclase (Murad et al., 1993). 1H$[1,2,4]$ oxadiazolo[4,3,-a $]$ quinoxaline-1-one (ODQ) is a specific and potent inhibitor of activation of soluble guanylyl cyclase by nitric oxide (Brunner et al., 1995). ODQ had no effect on DEA/NOinduced adenosine accumulation at $10 \mu \mathrm{M}(n=3$; data not shown), despite the fact that it completely prevented the increase in cGMP in cultures exposed to DEA/NO at $1 \mu \mathrm{M}$. As an alternative approach to test the involvement of cGMP in extracellular adenosine accumulation, we tested the effect of $1 \mathrm{~mm}$ 8-bromo-cGMP $(n=3)$ and 500-1000 $\mu \mathrm{M}$ 8-CPT-cGMP $(n=2)$, membrane-permeable, cyclic nucleotide phosphodiesterase-resistant cGMP analogs. These compounds produced no effect on extracellular adenosine accumulation (data not shown).

\section{Effect of DEA/NO on intracellular ATP content}

Nitric oxide inhibits the Krebs' cycle (Castro et al., 1998) and electron transport at both complex I and complex IV (Clementi et al., 1998) and has been shown to cause compromise of mitochondrial function in neurons (Brorson et al., 1999). These multiple effects on cellular energy metabolism might be expected to lead to ATP hydrolysis, intracellular adenosine accumulation, and extracellular adenosine accumulation mediated by adenosine transporters. To test this we determined the effect of DEA/NO on intracellular ATP content. Although in individual experiments the effect of $30 \mathrm{~min}$ exposure to $100 \mu \mathrm{M}$ DEA/NO did not always attain significance, in the pooled results of nine experiments that were performed, we found a significant $(p<0.05)$ fall in ATP content to $78 \pm 8 \%$ (22\% reduction) of control values (Fig. $5 \mathrm{~A})$. The effect of nitric oxide on ATP content that we observed was less than that reported previously (Brorson et al., 1999), but the culture paradigm and experimental procedure that we used were different. When we tested the effect of DEA/NO on ATP content in neuronal cultures without a $2 \mathrm{hr}$ preincubation period, we found that ATP content was reduced to $29 \pm 4 \%$ of control at $30 \mu \mathrm{M}$ and to $20 \pm 5 \%$ of control at $100 \mu \mathrm{M}$ (30 min exposure; $n=4)$, comparable to the magnitude of the effects reported in the previous study.

If the effect of DEA/NO on extracellular adenosine were mediated by accumulation of intracellular adenosine secondary to net ATP hydrolysis, then other causes of ATP depletion would also be expected to cause accumulation of extracellular adenosine. We therefore tested the effect of the complex I inhibitor rotenone, chosen because nitric oxide is an inhibitor of complex I. First, we found that rotenone, at a concentration of $100 \mathrm{nM}$, caused significant $(p<0.001)$ ATP hydrolysis, as did DEA/NO in these experiments $(p<0.001)$. Figure 5B shows data pooled from three experiments. In these experiments, in the presence of rotenone, ATP extracted from cultures was $66 \pm 7.9 \%$ of control. The effect of rotenone occluded the effect of nitric oxide; there was no additional loss of ATP with DEA/NO plus rotenone compared with rotenone alone, suggesting that the effect of rotenone might be attributable to inhibition of the same target as nitric oxide. Finally, we also tested the ability of rotenone to cause accumulation of extracellular adenosine. Rotenone very effectively increased extracellular adenosine, as shown in Figure $5 C$. In this particular representative experiment, we determined an $\mathrm{EC}_{50}$ value of $29 \mathrm{~nm}$ for this effect of rotenone. In three experiments that were performed, the $\mathrm{EC}_{50}$ was $17.2 \pm 11 \mathrm{~nm}$.

\section{Nitric oxide inhibits adenosine kinase}

The previous experiments showed that nitric oxide induced release of adenosine itself from neurons by a cGMP-independent mechanism and that exposure to nitric oxide was associated with a decrease in intracellular ATP content. Adenosine kinase is a critical enzyme in the regulation of intracellular and extracellular adenosine concentration (Arch and Newsholme, 1978; Lloyd and Fredholm, 1995). Adenosine kinase has the interesting property that it is inhibited by its substrate, adenosine, at concentrations not far above the $K_{\mathrm{m}}$ for that substrate (Miller et al., 1979; Palella et al., 1980; Rottlan and Miras Portugal, 1985; McNally et al., 1997; Mathews et al., 1998). Therefore, we asked the question whether inhibition of adenosine kinase activity might be occurring as a result of exposure to nitric oxide, hydrolysis of ATP, and increase in intracellular adenosine levels.

We assayed adenosine kinase activity by an established method (Lynch et al., 1998), exposing cultures to ${ }^{14} \mathrm{C}$-adenosine, taking lysates at appropriate times, and counting radioactivity that remained associated with ion-exchange filters, a property possessed by the phosphorylated but not parent compound. Neuronal cultures incubated with ${ }^{14} \mathrm{C}$-adenosine demonstrated an increase in radioactivity binding to the filters that was blocked by the presence of 5 '-iodotubericidin, a specific inhibitor of adenosine kinase (Lynch et al., 1998). Figure $6 A$ shows a representative experiment examining the concentration dependence of the effect of $5^{\prime}$ iodotubericidin on adenosine kinase activity. In three experiments, $5^{\prime}$-iodotubericidin blocked adenosine kinase activity with an $\mathrm{IC}_{50}$ of $4.3 \pm 4.5 \mathrm{~nm}$. To test the effect of nitric oxide on adenosine kinase activity, cultures were pretreated with $100 \mu \mathrm{M}$ DEA/NO for $5 \mathrm{~min}$ before addition of radioactivity. In this case, the adenosine kinase activity was almost completely inhibited for the first $5 \mathrm{~min}$ (zero counts above background present at the 5 min time point), after which some activity was detectable in the next $15 \mathrm{~min}$. Figure $6 B$ shows a representative experiment of three that were performed. Between 20 and $30 \mathrm{~min}$, the radioactivity on the filters increased at a nearly normal rate, indicating recovery of adenosine kinase activity.

If a rise in intracellular adenosine attributable to inhibition of ATP synthesis by nitric oxide was responsible for the inhibition of adenosine kinase activity, we reasoned that another cause of inhibition of ATP synthesis would have a similar effect. Therefore we tested the effect of rotenone on adenosine kinase activity. We found that this was almost completely inhibited by the presence of $100 \mathrm{~nm}$ rotenone, consistent with a mechanism whereby accumulation of intracellular adenosine inhibits adenosine kinase activity. 

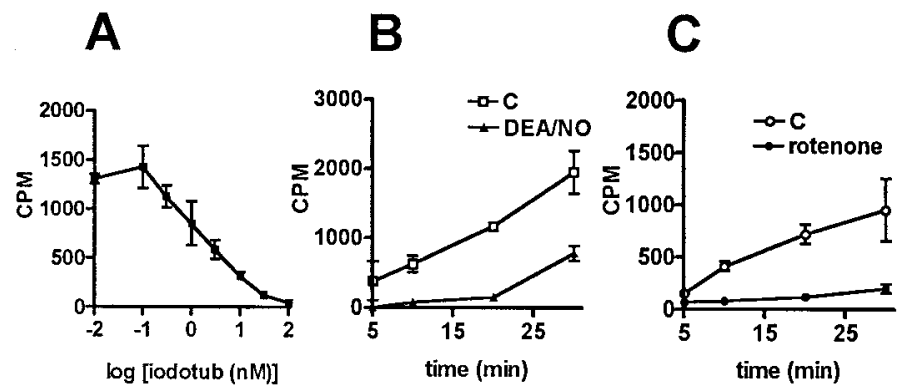

Figure 6. Effect of $5^{\prime}$-iodotubericidin (IT), DEA/NO, and rotenone on adenosine kinase activity. $A$, Neuronal cultures were incubated with selected concentrations of $5^{\prime}$-iodotubericidin (iodotub) for $5 \mathrm{~min}$ and then exposed to ${ }^{14} \mathrm{C}$-adenosine. After $15 \mathrm{~min}$, lysate was prepared and filtered, and radioactivity bound to filters was assayed. Radioactivity bound to filters was nearly completely eliminated at $100 \mathrm{nM}$ IT. The experiment shown is representative of three experiments that were performed. $B$, Cultures were pretreated for 5 min with $100 \mu \mathrm{M}$ DEA/NO and then ${ }^{14} \mathrm{C}$-adenosine was added. Samples were taken at 5, 10, 20, and $30 \mathrm{~min}$. Adenosine kinase activity was markedly suppressed compared with control (vehicle only) for the first $20 \mathrm{~min}$ after exposure to radioactivity, after which the activity increased steeply. The experiment shown is representative of three experiments that were performed. $C$, Cultures were pretreated with rotenone $(100 \mathrm{nM})$ or no drug for $5 \mathrm{~min}$, after which they were exposed to ${ }^{14} \mathrm{C}$ adenosine, and samples were prepared at selected times and assayed by filter binding. Rotenone at this concentration effectively suppressed the activity of adenosine kinase. The experiment shown is representative of three that were performed.

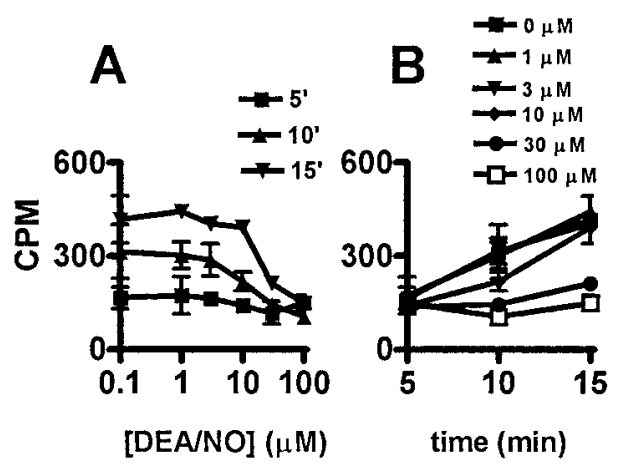

Figure 7. Concentration and time dependence of the effect of DEA/NO on adenosine kinase activity. Cultures were exposed to selected concentrations of DEA/NO before addition of radioactivity. Samples were taken at 5,10 , and 15 min after addition of radioactivity. The same data are plotted in $A$, with concentration as the independent variable, and in $B$ with time as the independent variable. $A$, Clear concentration dependence is evident at the 10 and $15 \mathrm{~min}$ intervals. $B$, The accumulation of radioactivity appears to be linear over $15 \mathrm{~min}$ in the absence of DEA/NO. The experiment shown is representative of three experiments that were performed.

Figure $6 C$ is representative of three experiments that were performed.

Neuronal cultures were exposed to selected concentrations of DEA/NO up to $100 \mu \mathrm{M}$ for 5,10 , or 15 min intervals to determine the variation of activity with concentration. Figure $7, A$ and $B$, shows results from a single representative experiment of three that were performed. Figure $7 A$ shows the concentration dependence of the adenosine kinase activity represented by a family of curves, each obtained at a different time interval after addition of radioactivity $(5,10$, and $15 \mathrm{~min})$. In Figure $7 B$, the same data are plotted to show the time dependence of uptake at different concentrations. In three experiments we determined an $\mathrm{IC}_{50}$ value for $\mathrm{DEA} / \mathrm{NO}$ on adenosine kinase activity of $10.7 \pm 5.9 \mu \mathrm{M}$, using the data obtained at $10 \mathrm{~min}$, and a value of $20.1 \pm 6.6 \mu \mathrm{M}$ using the data obtained at $15 \mathrm{~min}$. These values are not significantly different.

To test whether the effect of DEA/NO on adenosine kinase activity was caused by nitric oxide, experiments were performed in which hemoglobin or carboxy-PTIO (a water-soluble derivative of PTIO) were used to scavenge nitric oxide. Figure 8 is a representative experiment of three that were performed. There was no

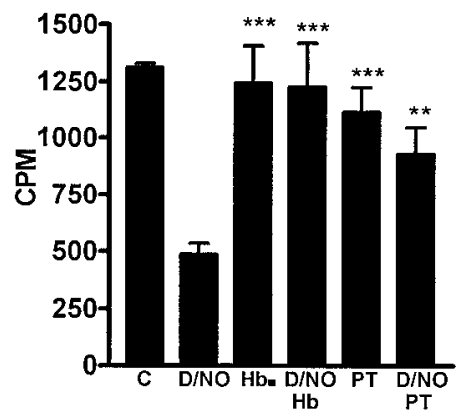

Figure 8. Effect of scavengers of nitric oxide on the effect of DEA/NO on adenosine kinase activity. Cultures were exposed to DEA/NO $(D / N O)$, hemoglobin $(H b)$, or carboxy PTIO $(P T)$, or DEA/NO plus an inhibitor, followed by addition of radioactivity after $5 \mathrm{~min}$. Exposure was performed for another $10 \mathrm{~min}$, after which lysate was prepared and filtered, and radioactivity bound to filters was assayed. Hemoglobin completely blocked the effect of DEA/NO: there was no significant difference between the effect of $\mathrm{Hb}$ alone and $\mathrm{DEA} / \mathrm{NO}$ plus $\mathrm{Hb}$. There was also no significant difference between carboxy PTIO by itself and carboxy PTIO plus DEA/ NO. The statistics indicated represent the significance of the difference between the experimental condition under the asterisks and the $\mathrm{D} / \mathrm{NO}$ condition. The experiment shown is representative of three experiments that were performed.
A

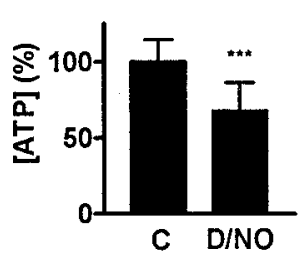

B

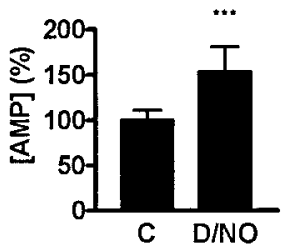

C

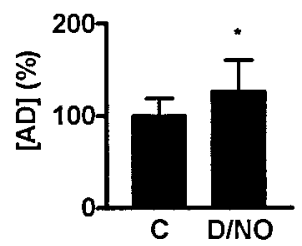

Figure 9. Effect of DEA/NO on intracellular ATP, AMP, and adenosine content. Neuronal cultures were exposed to DEA/NO $(D / N O, 100 \mu \mathrm{M})$ for $5 \mathrm{~min}$. Cell extracts were prepared by adding $20 \mathrm{~mm}$ sodium acetate and 2 mM EDTA to the cultures, freezing on dry ice, and thawing. Data were normalized by dividing individual values by the control mean for each experiment. Graphs represent data pooled from three separate experiments. $A$, Change in intracellular ATP concentration; $p<0.001 . B$, Change in intracellular AMP concentration; $p<0.001$. $C$, Change in intracellular adenosine concentration; $p<0.05$.

difference between the cultures exposed to DEA/NO plus hemoglobin and cultures exposed to hemoglobin alone, or between cultures exposed to DEA/NO plus carboxy-PTIO and cultures exposed to carboxy-PTIO alone. Therefore, the effect of DEA/NO on adenosine kinase activity is mediated by nitric oxide, as we have shown for the effect of DEA/NO on extracellular adenosine accumulation.

Finally, we tested the hypothesis that nitric oxide caused the inhibition of adenosine kinase by increasing intracellular adenosine concentrations. Cultures exposed to DEA/NO for $5 \mathrm{~min}$ were extracted, and the extracts were assayed by HPLC. Figure 9 shows data pooled from three experiments. ATP concentration decreased in DEA/NO-treated cultures compared with control cultures $(70 \pm$ $15 ; p<0.0001)$. AMP concentration increased in treated cultures $(150 \pm 24 \% ; p<0.0001)$, as did adenosine concentration $(125 \pm$ $18 \% ; p<0.01)$.

\section{DISCUSSION}

We here present the following evidence that nitric oxide causes an increase in extracellular adenosine in neuronal cultures: (1) different donors all produced a similar effect; (2) nitric oxide scavengers blocked the increase in adenosine; and (3) the product of hydrolysis of DEA/NO, diethylamine, had no effect.

Other publications have presented direct or indirect evidence that nitric oxide stimulates adenosine release from cells and tissue derived from the CNS (Boulton et al., 1994; Broome et al., 1994; Fischer et al., 1995; Fallahi et al., 1996). However, Craig and White 
(1993) found no effect on adenosine of the nitric oxide donor sodium nitroprusside in superfused rat cortical slices. They also found no evidence to suggest that NMDA-stimulated adenosine accumulation was mediated by activation of nitric oxide synthase and release of nitric oxide. Finally, in a recent study of the regulation of adenosine in rat striatum using high-energy focused microwave irradiation to preserve postmortem tissue levels, it was found that intrastriatal arginine decreased and the inhibitor of nitric oxide synthase, NAME, increased basal adenosine levels, results opposite to those expected on the basis of the present findings. Consistent with the findings of Craig and White (1993) mentioned above, neither arginine nor NAME had any effect on the NMDA-stimulated increase in adenosine (Delaney et al., 1998). In unpublished studies from our own laboratory, we found that NMDA stimulated extracellular adenosine accumulation in neuronal cultures and that inhibitors of nitric oxide synthase did not block this effect. In these cultures a very small number of neurons stain positively for nitric oxide synthase $(\sim 1 \%)$, and therefore the lack of effect may be attributable to the low levels of nitric oxide production by the few NOS-containing neurons that are present (Samdani et al., 1997)

The present report strongly suggests that adenosine accumulation in neuronal cultures evoked by nitric oxide does not derive from extracellular AMP via the action of $5^{\prime}$-ectonucleotidase, because its accumulation could not be blocked to any degree by GMP, an inhibitor of 5'-ectonucleotidase (Pearson, 1987). Although the adenosine appears to derive from adenosine transported across the plasma membrane rather than from the transport and extracellular degradation of a nucleotide, it was not possible to block the accumulation using inhibitors of adenosine transport. Therefore, release could be occurring via an inhibitor-insensitive equilibrative transporter or one or more of the inhibitor-insensitive concentrative transporters, either because intracellular adenosine concentration had built up in excess of what the transport system could maintain or because the sodium gradient had diminished, for example because of a compromise in energy metabolism by nitric oxide (Thorn and Jarvis, 1996). In fact, iodoacetate-evoked release of adenosine by a concentrative transporter has been observed (Foga et al., 1996).

Although the best-known action of nitric oxide is its ability to activate guanylyl cyclase, a novel finding of this report is that the action of nitric oxide on extracellular adenosine accumulation appears to be independent of cGMP metabolism. Thus, we found that cGMP analogs did not mimic the effect of nitric oxide and an inhibitor of guanylyl cyclase activation did not block the effects of nitric oxide. Other actions of nitric oxide are known and include interaction with heme and non-heme iron in various proteins, as well as $S$-nitrosylation reactions (Stamler et al., 1997). In addition, nitric oxide rapidly interacts with superoxide to form peroxynitrite (Koppenol et al., 1992). However, because peroxynitrite formation is blocked by superoxide dismutase, and we found no effect of inclusion of superoxide dismutase (Fig. 1), it seems likely that the effect of nitric oxide donors on adenosine accumulation that we observed is caused by nitric oxide itself and not peroxynitrite. Our experiments do not exclude the possibility that peroxynitrite itself may stimulate adenosine formation, but because of its neurotoxicity and the confounding effects of leakage from damaged membranes, we have not pursued this possibility in the present work.

From previous work (Clementi et al., 1998; Brorson et al., 1999), it was expected that nitric oxide would cause ATP concentration derived from oxidative phosphorylation to rapidly fall, leading to an accumulation of AMP and adenosine. To pursue this, we measured ATP extractable from neuronal cultures after exposure to DEA/NO and found a significant decrease in ATP content compared with control. This reduction in ATP was not observed in the presence of hemoglobin (data not shown). As a test of the notion that this metabolic blockade might be sufficient for the extracellular adenosine accumulation that was observed, we tested the effect of rotenone, a complex I inhibitor, and found that it potently stimulated adenosine release in addition to decreasing intracellular ATP content.

Adenosine kinase is a critical enzyme in intracellular adenosine metabolism. Interestingly, adenosine kinase is inhibited by adenosine at a concentration not far from the $\mathrm{Km}$ for its catalytic activity (Miller et al., 1979; Palella et al., 1980; Rotllan and Miras Portugal, 1985; Hawkins and Bagnara, 1987; McNally et al., 1997; Mathews et al., 1998). Exposure to nitric oxide or any other experimental manipulation that causes intracellular adenosine accumulation would potentially inhibit adenosine kinase, resulting in further adenosine accumulation. Indeed, another novel observation reported here is that exposure of neurons to nitric oxide was associated with inhibition of adenosine kinase. Thus, we found that 100 $\mu \mathrm{M}$ DEA/NO caused a nearly complete suppression of adenosine kinase activity in neuronal cultures. Similar to what was observed with DEA/NO, rotenone also inhibited adenosine kinase activity. Therefore, it appears that the effects of DEA/NO and rotenone on intracellular ATP hydrolysis and consequent elevation in intracellular adenosine levels might account for the blockade in adenosine kinase that was observed; however, a direct action of either nitric oxide or rotenone on adenosine kinase cannot be excluded.

Although the change in the intracellular adenosine that was measured was small, given the presence in the plasma membrane of equilibrative transporters, it is perhaps remarkable that we were able to measure any increase. The change in adenosine that we measured was averaged over whole cells and over all the cells that were present. Some cells may be more affected than others, and within a given cell, compartmentalization of intracellular adenosine increases might be important-for example, in the vicinity of mitochondria affected by nitric oxide.

Other possibilities need to be considered as well, however, including the involvement of a second messenger system, such as protein kinase C (Sinclair et al., 2000), and a direct effect of lowering of the concentration of ATP, which provides the phosphate group and the energy for the phosphorylation of adenosine by adenosine kinase. However, normal levels of ATP are well above saturation at the ATP binding site (McNally et al., 1997), and a change of $\sim 20 \%$ in cellular ATP, as we have observed, is not likely to be responsible for any reduction of activity.

Adenosine kinase inhibition has also been demonstrated in other settings associated with ATP hydrolysis: hypoxia in the guinea pig heart (Decking et al., 1997) and oxygen-glucose deprivation in rat cortical neuronal cultures (Lynch et al., 1998). The consequence of inhibition of adenosine kinase activity, triggered by a small increase in intracellular adenosine concentration, is that cells are switched into a mode of extracellular adenosine accumulation. Thus adenosine kinase is likely to be of central importance in the protective role of adenosine in the setting of energy compromise. In addition, the switching property of adenosine kinase may be of physiological importance as well, for example in the production of sleep.

Because of the high expression of NOS in neurons of the LDT/ PPT, it seems likely that nitric oxide is important in the function of this nucleus. Inhibition of NOS in general has been shown to result in a diminution in sleep, whereas electrophysiological studies are consistent with a facilitatory effect of nitric oxide on arousal and REM generation mechanisms in target areas of the LDT/PPT. We think that this paradox might be resolvable by considering the effects of nitric oxide as both phasic and tonic. Acute release of nitric oxide by the activity of NOS-containing neurons has potentiating effects on the release of neurotransmitters such as acetylcholine (Leonard and Lydic, 1997) and glutamate (Montague et al., 1994; Kara and Friedlander, 1999) and increases $I_{\mathrm{H}}$ to change the firing pattern of thalamic neurons, all of which effects act to promote the arousal or REM generation function of the LDT/PPT. Sustained, tonic activity of nitrergic neurons may produce accumulation of extracellular adenosine resulting in an increasing drive to sleep. Our hypothesis is that the activity of LDT/PPT neurons during waking is associated with the continuous release of nitric oxide in sleep-relevant target areas, leading to the accumulation of 
adenosine in these areas and an increasing tendency to sleep. It has been generally thought that the release of adenosine that occurs with waking may be attributable to a nonspecific depletion of energy stores during waking (Benington and Heller, 1995). Our view is that this may be attributable rather to a specific metabolic action of the nitric oxide that is released coincidentally with the activity of neurons that play a central role in arousal. We postulate that release of adenosine by the action of nitric oxide on cellular energy production may be important in homeostatic sleep mechanisms.

\section{REFERENCES}

Arch JR, Newsholme EA (1978) The control of the metabolism and the hormonal role of adenosine. Essays Biochem 14:82-123.

Baldwin SA, Mackey JR, Cass CE, Young JD (1999) Nucleoside transporters: molecular biology and implications for therapeutic development. Mol Med Today 5:216-224.

Barber R, Butcher RW (1983) The egress of cyclic AMP from metazoan cells. Adv Cyclic Nucleotide Res 15:119-138.

Benington JH, Heller HC (1995) Restoration of brain energy metabolism as the function of sleep. Prog Neurobiol 45:347-360.

Boulton CL, Irving AJ, Southam E, Potier B, Garthwaite J, Collingridge GL (1994) The nitric oxide-cyclic GMP pathway and synaptic depression in rat hippocampal slices. Eur J Neurosci 6:1528-1535.

Broome MR, Collingridge GL, Irving AJ (1994) Activation of the NOcGMP signalling pathway depresses hippocampal synaptic transmission through an adenosine receptor-dependent mechanism. Neuropharmacology 33:1511-1513.

Brorson JR, Schumacker PT, Zhang H (1999) Nitric oxide acutely inhibits neuronal energy production. J Neurosci 19:147-158.

Brunner F, Stessel H, Kukovetz WR (1995) Novel guanylyl cyclase inhibitor, ODQ, reveals role of nitric oxide, but not of cyclic GMP in endothelin-1 secretion. FEBS Lett 376:262-266.

Burlet S, Cespuglio R (1997) Voltammetric detection of nitric oxide (NO) in the rat brain: its variations throughout the sleep-wake cycle. Neurosci Lett 226:131-135.

Burlet S, Leger L, Cespuglio R (1999) Nitric oxide and sleep in the rat: a puzzling relationship. Neuroscience 92:627-639.

Castro LA, Robalinho RL, Cayota A, Meneghini R, Radi R (1998) Nitric oxide and peroxynitrite-dependent aconitase inactivation and ironregulatory protein-1 activation in mammalian fibroblasts. Arch Biochem Biophys 359:215-224.

Cespuglio R, Burlet S, Faradji-Prevautel H (1998) 5-hydroxyindoles compounds and nitric oxide voltammetric detection in the rat brain: changes occurring throughout the sleep-wake cycle. J Neural Transm 105:205-215.

Clementi E, Brown GC, Feelisch M, Moncada S (1998) Persistent inhibition of cell respiration by nitric oxide: crucial role of $S$-nitrosylation of mitochondrial complex I and protective action of glutathione. Proc Natl Acad Sci USA 95:7631-7636.

Craig CG, White TD (1993) NMDA-evoked adenosine release from rat cortex does not require the intermediate formation of nitric oxide. Neurosci Lett 158:167-169.

Decking UK, Schlieper G, Kroll K, Schrader J (1997) Hypoxia-induced inhibition of adenosine kinase potentiates cardiac adenosine release. Circ Res 81:154-164.

Delaney SM, Shepel PN, Geiger JD (1998) Levels of endogenous adenosine in rat striatum. I. Regulation by ionotropic glutamate receptors, nitric oxide and free radicals. J Pharmacol Exp Ther 285:561-567.

Dzoljic MR, De Vries R, Van Leeuwen R (1996) Sleep and nitric oxide: effects of 7-nitro indazole, inhibitor of brain nitric oxide synthase. Brain Res 718:145-150.

Fallahi N, Broad RM, Jin S, Fredholm BB (1996) Release of adenosine from rat hippocampal slices by nitric oxide donors. J Neurochem 67:186-193.

Fischer H, Prast H, Philippu A (1995) Adenosine release in the ventral striatum of the rat is modulated by endogenous nitric oxide. Eur J Pharmacol 275:R5-6.

Foga IO, Geiger JD, Parkinson FE (1996) Nucleoside transportermediated uptake and release of $\left[{ }^{3} \mathrm{H}\right] \mathrm{L}$-adenosine in $\mathrm{DDT}_{1} \mathrm{MF}-2$ smooth muscle cells. Eur J Pharmacol 318:455-460.

Furchgott RF (1998) Nitric oxide: from basic research on isolated blood vessels to clinical relevance in diabetes. An R Acad Nac Med (Madr) 115:317-331.

Furchgott RF, Zawadzki JV (1980) The obligatory role of endothelial cells in the relaxation of arterial smooth muscle by acetylcholine. Nature 288:373-376.

Hawkins CF, Bagnara AS (1987) Adenosine kinase from human erythrocytes: kinetic studies and characterization of adenosine binding sites. Biochemistry 26:1982-1987.

Kapas L, Fang J, Krueger JM (1994a) Inhibition of nitric oxide synthesis inhibits rat sleep. Brain Res 664:189-196.
Kapas L, Shibata M, Kimura M, Krueger JM (1994b) Inhibition of nitric oxide synthesis suppresses sleep in rabbits. Am J Physiol 266:R151-R157.

Kara P, Friedlander MJ (1999) Arginine analogs modify signal detection by neurons in the visual cortex. J Neurosci 19:5528-5548.

Koppenol WH, Moreno JJ, Pryor WA, Ischiropoulos H, Beckman JS (1992) Peroxynitrite, a cloaked oxidant formed by nitric oxide and superoxide. Chem Res Toxicol 5:834-842.

Leonard TO, Lydic R (1997) Pontine nitric oxide modulates acetylcholine release, rapid eye movement sleep generation, and respiratory rate. J Neurosci 17:774-785.

Li Y, Rosenberg PA (1998) Nitric oxide evokes extracellular adenosine accumulation in cultures of rat forebrain neurons. Soc Neurosci Abstr 24:2059.

Lipton SA, Choi Y-B, Pan Z-H, Lei SZ, Shen H-SV, Sucher NJ, Loscalzo J, Singel DJ, Stamler JS (1993) A redox-based mechanism for the neuroprotective and neurodestructive effects of nitric oxide and related nitroso-compounds. Nature 364:626-632.

Lloyd HGE, Fredholm BB (1995) Involvement of adenosine deaminase and adenosine kinase in regulating extracellular adenosine concentration in rat hippocampal slices. Neurochem Int 26:387-395.

Lynch III JJ, Alexander KM, Jarvis MF, Kowaluk EA (1998) Inhibition of adenosine kinase during oxygen-glucose deprivation in rat cortical neuronal cultures. Neurosci Lett 252:207-210.

MacDonald WF, White TD (1985) Nature of extrasynaptosomal accumulation of endogenous adenosine evoked by $\mathrm{K}+$ and veratridine. J Neurochem 45:791-797.

Maeda H, Akaike T, Yoshida M, Sato K, Noguchi Y (1995) A new nitric oxide scavenger, imidazolineoxyl $\mathrm{N}$-oxide derivative, and its effects in pathophysiology and microbiology. Curr Top Microbiol Immunol 196:37-50.

Maragos CM, Morley D, Wink DA, Dunams TM, Saavedra JE, Hoffman A, Bove AA, Isaac L, Hrabie JA, Keefer LK (1991) Complexes of NO with nucleophiles as agents for the controlled biological release of nitric oxide. Vasorelaxant effects. J Med Chem 34:3242-3247.

Mathews II, Erion MD, Ealick SE (1998) Structure of human adenosine kinase at 1.5 A resolution. Biochemistry 37:15607-15620.

McNally T, Helfrich RJ, Cowart M, Dorwin SA, Meuth JL, Idler KB, Klute KA, Simmer RL, Kowaluk EA, Halbert DN (1997) Cloning and expression of the adenosine kinase gene from rat and human tissues. Biochem Biophys Res Commun 231:645-650.

Miller RL, Adamczyk DL, Miller WH (1979) Adenosine kinase from rabbit liver. I. Purification by affinity chromatography and properties. J Biol Chem 254:2339-2345.

Montague PR, Gancayco CD, Winn MJ, Marchase RB, Friedlander MJ (1994) Role of NO production in NMDA receptor-mediated neurotransmitter release in cerebral cortex. Science 263:973-977.

Mooradian DL, Hutsell TC, Keefer LK (1995) Nitric oxide (NO) donor molecules: effect of NO release rate on vascular smooth muscle cell proliferation in vitro. J Cardiovasc Pharmacol 25:674-678.

Murad F, Forstermann U, Nakane M, Pollock J, Tracey R, Matsumoto T, Buechler W (1993) The nitric oxide-cyclic GMP signal transduction system for intracellular and intercellular communication. Adv Second Messenger Phosphoprotein Res 28:101-109.

Palella TD, Andres CM, Fox IH (1980) Human placental adenosine kinase. Kinetic mechanism and inhibition. J Biol Chem 255:5264-5269.

Pape H-C, Mager R (1992) Nitric oxide controls oscillatory activity in thalamocortical neurons. Neuron 9:441-448.

Pearson JD (1987) Ectonucleotidases. Measurement of activities and use of inhibitors. In: Methods in pharmacology (Paton DM, ed), pp 83-107. New York: Plenum.

Porkka-Heiskanen T, Strecker RE, Thakkar M, Bjorkum AA, Greene RW, McCarley RW (1997) Adenosine: a mediator of the sleep-inducing effects of prolonged wakefulness. Science 276:1265-1268.

Portas CM, Thakkar M, Rainnie DG, Greene RW, McCarley RW (1997) Role of adenosine in behavioral state modulation: a microdialysis study in the freely moving cat. Neuroscience 79:225-235.

Rainnie DG, Grunze HCR, McCarley RW, Greene RW (1994) Adenosine inhibition of mesopontine cholinergic neurons: implications for EEG arousal. Science 263:689-692

Rosenberg PA (1991) Accumulation of extracellular glutamate and neuronal death in astrocyte-poor cortical cultures exposed to glutamine. Glia 4:91-100.

Rosenberg PA, Dichter MA (1989) Extracellular cAMP accumulation and degradation in rat cerebral cortex in dissociated cell culture. J Neurosci 9:2654-2663.

Rosenberg PA, Li Y (1994) $\beta$-Adrenergic receptor-mediated regulation of extracellular adenosine in cerebral cortex in culture. $\mathrm{J}$ Neurosci 14:2953-2965

Rosenberg PA, Li Y (1995) Vasoactive intestinal peptide regulates extracellular adenosine levels in rat cortical cultures. Neurosci Lett 200:93-96.

Rosenberg PA, Li Y (1996) Forskolin evokes extracellular adenosine accumulation in rat cortical cultures. Neurosci Lett 211:49-52.

Rotllan P, Miras Portugal MT (1985) Adenosine kinase from bovine adrenal medulla. Eur J Biochem 151:365-371. 
Samdani AF, Newcamp C, Resink A, Facchinetti F, Hoffman BE, Dawson VL, Dawson TM (1997) Differential susceptibility to neurotoxicity mediated by neurotrophins and neuronal nitric oxide synthase. J Neurosci 17:4633-4641.

Schuman EM, Madison DV (1994) Nitric oxide and synaptic function. Annu Rev Neurosci 17:153-183.

Sinclair CJD, Shepel PN, Geiger JD, Parkinson FE (2000) Stimulation of nucleoside efflux and inhibition of adenosine kinase by $A_{1}$ adenosine receptor activation. Biochem Pharmacol 59:477-483.

Stamler JS, Toone EJ, Lipton SA, Sucher NJ (1997) (S)NO signals: translocation, regulation, and a consensus motif. Neuron 18:691-696.

Stone TW, Newby AC, Lloyd HGE (1990) Adenosine release. In: Adenosine and adenosine receptors (Williams M, ed), pp 173-224. Clifton, NJ: Humana.
Thorn JA, Jarvis SM (1996) Adenosine transporters. Gen Pharmacol 27:613-620.

Vincent SR, Kimura H (1992) Histochemical mapping of nitric oxide synthase in the rat brain. Neuroscience 46:755-784.

Wang GJ, Chung HJ, Schnuer J, Pratt K, Zable AC, Kavanaugh MP, Rosenberg PA (1998) High affinity glutamate transport in rat cortical neurons in culture. Mol Pharmacol 53:88-96.

Williams JA, Vincent SR, Reiner PB (1997) Nitric oxide production in rat thalamus changes with behavioral state, local depolarization, and brainstem stimulation. J Neurosci 17:420-427.

Yao SY, Ng AM, Muzyka WR, Griffiths M, Cass CE, Baldwin SA, Young JD (1997) Molecular cloning and functional characterization of nitrobenzylthioinosine (NBMPR)-sensitive (es) and NBMPR-insensitive (ei) equilibrative nucleoside transporter proteins (rENT1 and rENT2) from rat tissues. J Biol Chem 272:28423-28430. 